\title{
Effect of novel bicaval anastomosis technique for transplantation with and without prior cardiac surgery history
}

\author{
Feng Shi ${ }^{\#}$, Zongli Ren ${ }^{\#}$, Min Zhang ${ }^{\#}$, Zhiwei Wang^, Zhiyong Wu, Xiaoping Hu, Zhipeng Hu, \\ Hongbing Wu, Wei Ren, Luocheng Li, Yongle Ruan, Rui Hu \\ Department of Cardiovascular Surgery, Renmin Hospital of Wuhan University, Wuhan, China \\ Contributions: (I) Conception and design: Z Wang, F Shi; (II) Administrative support: Z Wang; (III) Provision of study materials or patients: Z Ren, \\ M Zhang, Z Wu, X Hu, Z Hu, H Wu, W Ren, L Li, Y Ruan, R Hu; (IV) Collection and assembly of data: F Shi, Z Ren, M Zhang; (V) Data analysis \\ and interpretation: F Shi, Z Ren, M Zhang; (VI) Manuscript writing: All authors; (VII) Final approval of manuscript: All authors. \\ \#These authors contributed equally to this work. \\ Correspondence to: Zhiwei Wang, PhD. Zhangzhidong Rd. 99, Wuchang District, Wuhan 430060, China. Email: wangzhiwei@whu.edu.cn.
}

Background: To evaluate the graft outcomes after orthotopic heart transplantation (HTx) with a novel bicaval anastomosis technique between recipients with and without a history of prior cardiac surgery.

Methods: Of 70 patients who underwent HTx with a novel four-corners traction bicaval anastomosis technique from August 2017 to November 2019, 60 recipients underwent the HTx procedure as their first cardiac surgery (group A), while 10 recipients underwent HTx after prior cardiac surgery (group B). Patients in the two groups were compared in terms of their preoperative baseline variables such as etiological categories, history of blood transfusion and panel reactive antibody (PRA), intraoperative operation time and blood infusion volume, postoperative treatment time, and complications such as acute rejection and 30-day mortality as well as survival rates.

Results: Preoperative variables were comparable in group A and group B except for the history of blood transfusion ( $0 \%$ vs. $90.0 \%, \mathrm{P}<0.001$, respectively); the level of PRA was $7.5 \% \pm 5.8 \%$ and $9.5 \% \pm 10.9 \%$ for group $\mathrm{A}$ and $\mathrm{B}$, respectively $(\mathrm{P}=0.583)$, but the time of the operation was nearly 1 hour longer for group $\mathrm{B}$ than group $\mathrm{A}$ (all $\mathrm{P}<0.05)$. No cases of left atrial thrombosis and donor heart distortion were observed in either group. Reoperation ( $1.7 \%$ vs. $10.0 \%, \mathrm{P}=0.267)$, infection $(0 \%$ vs. $10.0 \%, \mathrm{P}=0.142)$, other postoperative complications as well as the 30 -day mortality $(1.7 \%$ vs. $10.0 \%, \mathrm{P}=0.267)$, and postoperative survival rates (91.5\% vs. 90.0\%, $\mathrm{P}=0.805$ ) were comparable between the two groups (all $\mathrm{P}>0.05$ ).

Conclusions: Four-corner traction bicaval anastomosis combined with a continuous everting suture technique may result in approximately comparable prognoses for heart recipients with a history of cardiac surgery when compared with those without a history of cardiac surgery and this technique may reduce the incidence of left atrial thrombosis and distortion. Further follow-up of the long-term outcomes will be required to validate these results.

Keywords: Heart transplantation (HTx); prior cardiac surgery; bicaval anastomosis; survival

Submitted Jan 20, 2021. Accepted for publication Apr 12, 2021.

doi: $10.21037 / \mathrm{atm}-21-317$

View this article at: http://dx.doi.org/10.21037/atm-21-317

^ ORCID: Feng Shi, 0000-0003-0364-7902; Zhiwei Wang, 0000-0001-5643-9344. 


\section{Introduction}

Although many advances have been made in treating end-stage heart disease, orthotopic heart transplantation (HTx) remains the gold standard treatment at present (1). With the tremendous progress and improvement of HTx surgical technology, perioperative treatment, and immunosuppressive therapy, the rate of HTx worldwide is increasing rapidly, and the outcomes of HTx continue to improve (2). However, donor heart sources are limited, and the donor heart should be given to those recipients with a relatively small risk to achieve maximum benefit (3). Whether a recipient for HTx with a prior cardiac surgery history is at greater risk than someone without prior cardiac surgery is still under discussion, and there is no clear consensus (4-6). Moreover, the widely used bicaval anastomosis method in HTx still has some postoperative complications such as left atrial thrombosis and anastomotic hemorrhage (7), which is why we propose a novel fourcorner traction bicaval anastomosis combined with a continuous everting suture technique. In this study, we evaluate the graft outcomes after HTx with this novel anastomosis technique between recipients with and without a prior history of cardiac surgery to determine whether a history of cardiac surgery contributes to a poorer prognosis for HTx and whether this novel anastomosis technique can reduce complications after transplantation. We present the following article in accordance with the TREND reporting checklist (available at http://dx.doi.org/10.21037/atm-21-317).

\section{Methods}

\section{Selection of patients}

Clinical data were collected from the hospital database and Organ Transplant Registry System. From August 2017 to November 2019, a total of $76 \mathrm{HTx}$ procedures were performed in our medical center. We included 70 recipients who underwent simple HTx procedures for end-stage heart disease after excluding three cases of combined HTx with other organs, one case of HTx combined with lung tumor resection and two cases of preoperative combined multiple organ dysfunction syndromes (MODS). We divided the 70 recipients into two groups categorized by their history of prior cardiac surgery: recipients without a cardiac surgery history before HTx were defined as group A, while those transplanted after previous cardiac surgery were defined as group B. According to the above criteria, 60 patients were included in group A, while group B consisted of 10 patients. Of the 10 recipients in group B, 6 had undergone valve replacement or plastic surgery, 3 had undergone aortic surgery, and 1 had undergone surgical repair for congenital heart disease. The follow-up time ranged from 1 to 35 months or at the time of the recipient's death. The same medical team completed the HTx operation and hospitalization treatment plan of all patients in this study, and all recipients followed the same criteria for blood transfusion, airway extubation, and intensive care unit (ICU) exit indications. All trial protocols were conducted following the Declaration of Helsinki (as revised in 2013) and approved by the Human Research Ethics Committees of Renmin Hospital of Wuhan University (WDRY2020-K230) (Wuhan, China). Our study complies with the International Society for Heart and Lung Transplantation ethics statement. Written informed consent was obtained from all patients or their guardians.

\section{Pre-operative assessment}

All patients underwent laboratory examinations, including routine hematological, biochemical assessment, virus screening, blood type, panel reactive antibody (PRA), and complement dependent cytotoxicity (CDC). Plasma levels of aspartate aminotransferase (AST), alanine aminotransferase (ALT), and creatinine were measured to assess liver and renal function. PRA testing was performed by testing recipient serum for complement-mediated lytic activity in the presence of anti-human immunoglobulin and dithiothreitol. The CDC experiment was conducted by mixing recipient serum with donor lymphoid tissue, excluding the presence of autoantibodies by autologous serum crossmatch with recipient lymphocytes. The donorrecipient crossmatch by $\operatorname{IgG}$ was considered positive if there was evidence of lymphocyte cytotoxicity in the presence of dithiothreitol.

\section{Surgical procedure}

A novel modified four-corners traction bicaval anastomosis combined with a continuous everting suture technique invented by Professor Zhiwei Wang, the corresponding author of this paper was used for HTx in this study. This procedure required complete resection of the right atrium and the long superior vena cava (SVC) (above the level of the azygos vena opening). The posterior wall of the donor's heart left atrium was removed and trimmed into a quadrangular cuff according to the distance of the four 


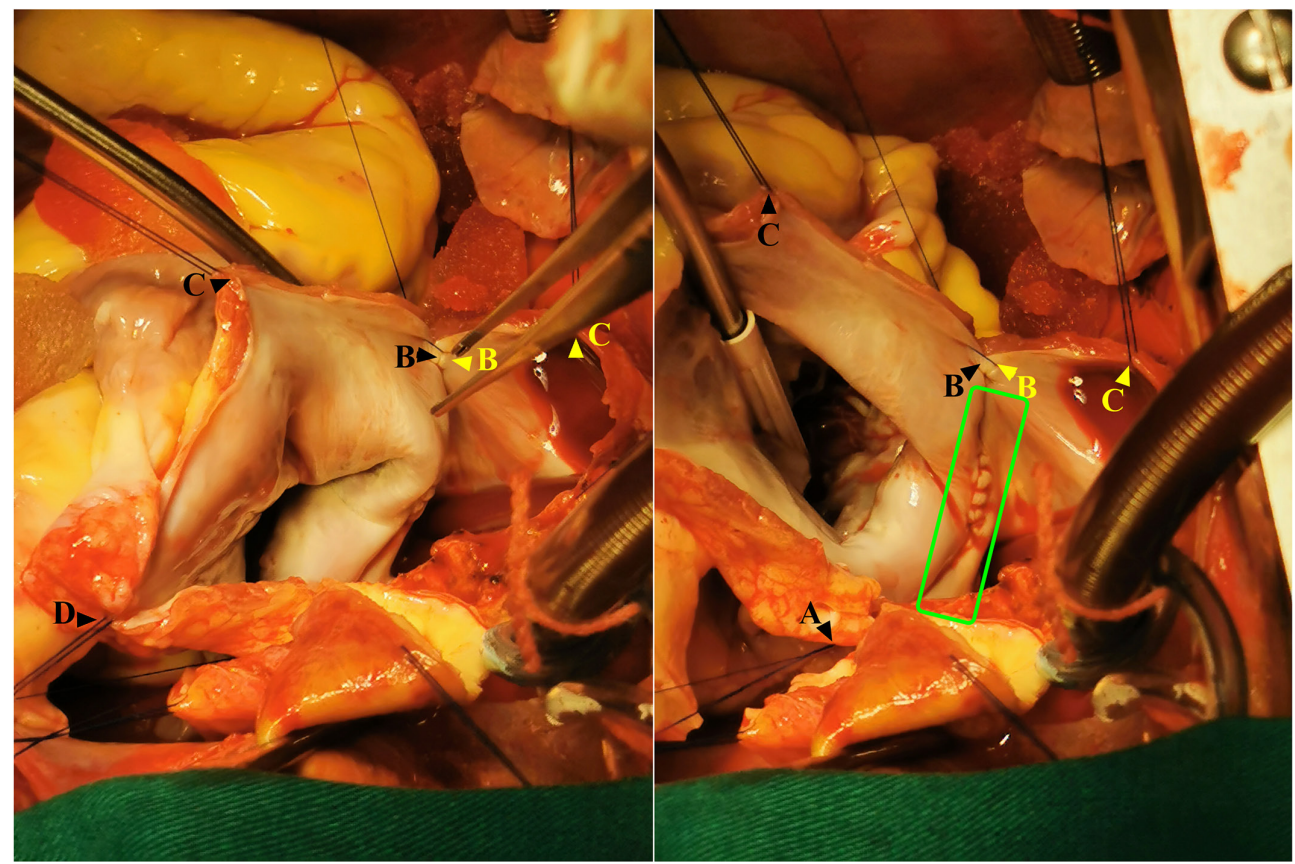

Figure 1 Four-corner traction bicaval anastomosis combined with a continuous everting suture technique. Four-corner traction divided the left atrial anastomosis into four shorter lines to easily adjust the distance for precise matching. Meanwhile, the integrity of the endocardium was maintained by the continuous everting suture along the four edges of left atrial cuff. Black arrows and letters indicated the four corners of the anastomosis in the left atrium of the donor heart. Yellow arrows and letters indicated the four corners of the left atrial cuff of recipient's heart. The contents of the green box show the internal view of the continuous everting suture.

pulmonary veins stoma and the size of the recipient's left atrium. The donor's heart was then perfused with a histidine-tryptophan-ketoglutarate (HTK) organ preservation solution and stored in a low-temperature environment of about $4{ }^{\circ} \mathrm{C}$. The recipient patient underwent a mid-sternal incision and then was intubated through the ascending aorta and the superior and inferior vena cava (IVC) to establish cardiopulmonary bypass (CPB). For recipients with a history of prior cardiac surgery, we used the femoral artery and vena cava cannulation to establish $\mathrm{CPB}$. The recipient's superior and IVC and ascending aorta were blocked, respectively. Then the recipient's heart was excised with a $2-3-\mathrm{cm}$ cuff left around the SVC and IVC. The left atrium incision of the donor heart was brought to the base of the recipient's left atrium, which had been removed leaving the posterior wall and small edges of the atrial cuff surrounding all four pulmonary veins. First, the four-corner traction technique of left atrial anastomosis was performed. The left superior and inferior corners of the recipient's left atrial cuff and the donor left atrial quadrilateral cuff adjacent to the left superior, and inferior pulmonary veins were sutured together with two stitches (point $\mathrm{A}$ and point $\mathrm{B}$ ). Then, the intersection of the inferior right corner of the donor left atrial quadrilateral cuff at the junction of the inferior edge of the left atrial quadrilateral cuff, the atrial septum and the left side of the IVC was marked with a retention suture (point C). Similarly, the intersection of the superior border of the quadrilateral cuff and the left side of the SVC indicated point $\mathrm{D}$, the superior right corner of the quadrilateral cuff of the donor left atrium and marked by another retaining suture. Left atrial anastomosis began with a complete continuous eversion suture from point $\mathrm{A}$ to $\mathrm{B}$. Then, $\mathrm{BC}, \mathrm{AD}$, and CD's boundaries were anastomosed in turn and knotted at each of the four points (Figure 1). Then the SVC and IVC were anastomosed by end-to-end and everted suture. Finally, the aorta and pulmonary artery were reconstructed in the usual way.

\section{Post-operative management and immunosuppression protocol}

When recipients returned to ICU after surgery, vasoactive 
drugs such as dopamine, epinephrine, nitroglycerin, etc., were administered intravenously according to circulating blood pressure when appropriate. The amount and color of the liquid in the draining tube of the mediastinal cavity after surgery was monitored as a condition for reoperation. Infectious complications were defined as a positive culture of bacteria from specimens taken from the respiratory tract, urinary, gastrointestinal, and other regions that caused corresponding organ dysfunction. Sensitive antibiotics were selected based on the bacterial culture at the site of infection. The immunosuppressive protocol was performed as follows: Recipients were routinely given basiliximab $20 \mathrm{mg}$ for immune induction 2 hours before surgery, which was then administered again on the 4th day after surgery. During the induction of anesthesia, a pulse-dose therapy of $500 \mathrm{mg}$ methylprednisolone was given, and the shock therapy of methylprednisolone was performed again after aortic de-clamping. After the operation, methylprednisolone was administered with a pulse-dose of $500 \mathrm{mg} / \mathrm{d}$ for 3 days. Tacrolimus was orally administered postoperatively with an approximate concentration of $8-10 \mathrm{ng} / \mathrm{mL}$, accompanied with mycophenolate mofetil $1,000 \mathrm{mg} / \mathrm{d}$, prednisone at $0.5 \mathrm{mg} / \mathrm{kg} / \mathrm{d}$ from the 4 th day postoperatively, then the dose of prednisone was gradually reduced to $10 \mathrm{mg} / \mathrm{d}$ and maintained.

\section{Statistical analysis}

Data were expressed as the mean \pm standard deviation for normally distributed continuous numerical variables, the median and interquartile range for non-normally distributed numerical variables, and as the frequency (percentage) for categorical variables. In comparisons of unmatched groups, the Student's $t$-test was applied to continuous variables, and the $\chi^{2}$ or Fisher's exact test was used for categorical variables. Survival was estimated using the Kaplan-Meier method and compared by the log-rank test. Differences were considered statistically significant when the two-sided $\mathrm{P}$ value was $<0.05$. All data were analyzed by SPSS software, version 27.0 (IBM Corp., Armonk, NY, USA).

\section{Results}

\section{Baseline characteristics}

Table 1 shows the demographics and baseline characteristics of the $60 \mathrm{HTx}$ recipients in group A and the 10 recipients in group B. The average level of PRA in group B was slightly higher than in group A, overall, no statistically significant differences were observed between the two groups in preoperative characteristics, including mean age, sex, body mass index (BMI), left ventricular ejection fraction (LVEF), creatinine, PRA, hypertension, diabetes, and diagnosis, as well as the mean follow-up range between the two groups (all $\mathrm{P}>0.05$ ). There was a significantly higher number of recipients with a blood transfusion history in group $\mathrm{B}$ than in group $\mathrm{A}(\mathrm{P}<0.001)$.

\section{Time of operation and intraoperative blood product transfusion}

The operation details and the number of blood products infused during the operation are presented in Table 2. The mean cold ischemia time was $155.0 \pm 18.8 \mathrm{~min}$ for group A compared to $267.6 \pm 94.4$ min for group B $(\mathrm{P}=0.004)$. The CPB time was $173.4 \pm 35.2 \mathrm{~min}$ for group A vs. $237.2 \pm$ $57.6 \mathrm{~min}$ for group $\mathrm{B}(\mathrm{P}=0.007)$. The mean aortic cross clamp (ACC) time was $90.5 \pm 10.6 v s .106 .6 \pm 22.0 \mathrm{~min}$ for groups $\mathrm{A}$ and $\mathrm{B}$, respectively $(\mathrm{P}=0.047)$. However, no statistical difference was observed in terms of intraoperative blood product infusion, including concentrated erythrocyte, platelet and plasma transfusion between the two groups.

\section{Postoperative early-term outcomes}

Postoperative early-term events are shown in Table 3. There was no statistical difference in terms of ICU treatment time, mechanical support, or postoperative early-term complications between the two groups. Only one case of postoperative acute rejection (AR) occurred in group $\mathrm{A}$, and there were postoperative atrial thrombosis cases observed in either group. The 30-day mortality was $1.7 \%$ vs. $10.0 \%$ for groups $\mathrm{A}$ and $\mathrm{B}$, respectively $(\mathrm{P}=0.267)$, and the last LVEF before discharge was $57.5 \% \pm 3.6 \%$ for group A vs. $58.2 \% \pm 2.7 \%$ for group B $(\mathrm{P}=0.559)$. Postoperative computed tomography angiography (CTA) illustrated an ideal and physiologically normal morphological structure of the left atrial and pulmonary veins without significant kinking, distortion or dilation (Figure 2).

\section{Postoperative mid-term survival analysis}

The postoperative mid-term survival is presented in Table 4 and Figure 3. The 1-year survival rates were $91.5 \%$ 
Table 1 Preoperative characteristics

\begin{tabular}{|c|c|c|c|}
\hline Parameters & Group A $(n=60)$ & Group B $(n=10)$ & $P$ value \\
\hline Male & 44 (73.3) & $7(70.0)$ & 0.869 \\
\hline $\mathrm{BMI}\left(\mathrm{kg} / \mathrm{m}^{2}\right)$ & $21.6 \pm 2.4$ & $21.0 \pm 2.4$ & 0.467 \\
\hline \multicolumn{4}{|l|}{ Echocardiography } \\
\hline LAD (mm) & $43.1 \pm 6.1$ & $60.6 \pm 12.8$ & 0.002 \\
\hline LVDD (mm) & $69.8 \pm 15.4$ & $65.6 \pm 13.4$ & 0.420 \\
\hline MR (grade 0-4) & $2.9 \pm 1.1$ & $1.4 \pm 1.8$ & 0.028 \\
\hline TR (grade 0-4) & $2.3 \pm 1.3$ & $2.0 \pm 1.6$ & 0.515 \\
\hline Cardiomyopathy & $39(65.0)$ & $5(50.0)$ & 0.579 \\
\hline Valvular disease & $3(5.0)$ & $4(40.0)$ & 0.004 \\
\hline Congenital heart disease & $0(0.0)$ & $1(10.0)$ & 0.143 \\
\hline $\operatorname{ALT}(\mathrm{U} / \mathrm{L})$ & $34.4 \pm 21.4$ & $50.6 \pm 55.7$ & 0.386 \\
\hline AST (U/L) & $33.7 \pm 16.2$ & $54.8 \pm 57.3$ & 0.276 \\
\hline $\mathrm{Cr}(\mathrm{mmol} / \mathrm{L})$ & $90.2 \pm 32.3$ & $84.2 \pm 51.5$ & 0.728 \\
\hline PRA (\%) & $7.5 \pm 5.8$ & $9.5 \pm 10.9$ & 0.583 \\
\hline Blood transfusion history & $0(0.0)$ & $9(90.0)$ & 0.000 \\
\hline History of CPR & $3(5.0)$ & $1(10.0)$ & 0.468 \\
\hline Mean follow-up range (months) & $18.6 \pm 8.4$ & $14.0 \pm 8.6$ & 0.115 \\
\hline
\end{tabular}

Values are expressed as mean \pm standard deviation or $n(\%)$. BMI, body mass index; LVEF, left ventricular ejection fraction; LAD, left atrium dimension; LVDD, left ventricular end diastolic dimension; MR, mitral regurgitation; TR, tricuspid regurgitation; ALT, alanine aminotransferase; AST, aspartate aminotransferase; $\mathrm{Cr}$, creatinine; PRA, panel reactive antibody; CPR, cardiopulmonary resuscitation.

(95\% CI: 80.7-96.4\%) for patients in group A and 90.0\% (95\% CI: 47.3-98.5\%) for group B. The 2- and 3-year survival rates for both groups were the same as the 1-year survival rate because there was no recipient death during the follow-up period from the second to the third postoperative year. There was no statistically significant difference in the patients' mid-term survival between the two groups $(\mathrm{P}=0.805)$.

\section{Discussion}

\section{Mediastinal adbesion prolongs operation time}

Due to the thoracotomy performed in the prior cardiac surgery, the adhesion between the heart and sternum was severe, which resulted in greater surgical difficulty and a longer tissue separation time (8). This is the reason for the prolonged operation time. As organ ischemia and 
Table 2 Intraoperative events

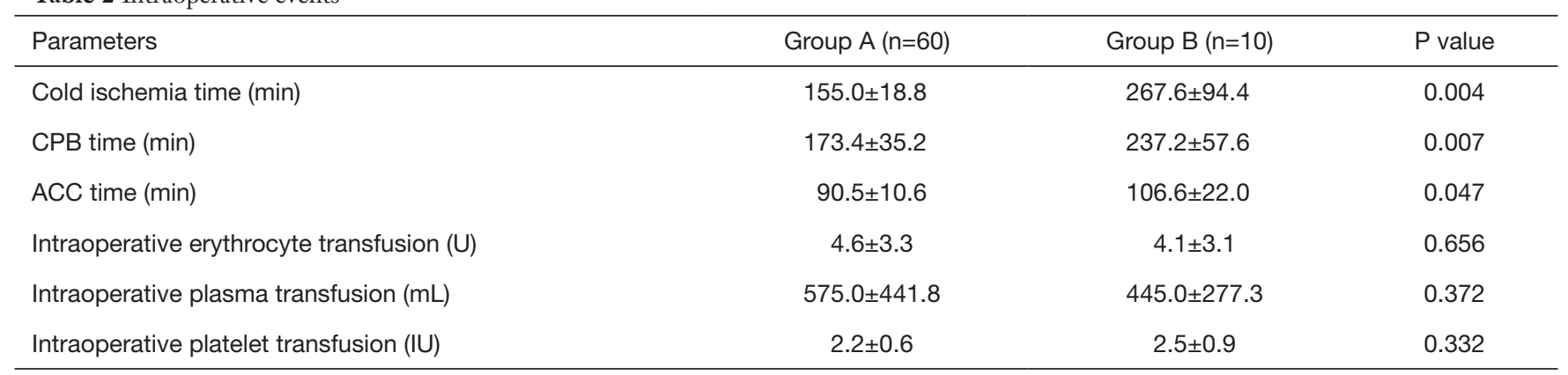

Values are expressed as mean \pm standard deviation. CPB, cardiopulmonary bypass; ACC, aortic cross clamp.

Table 3 Post-operative events

\begin{tabular}{|c|c|c|c|}
\hline Parameters & Group A $(n=60)$ & Group B $(n=10)$ & $P$ value \\
\hline Mechanical ventilation time (hours) & $10.1 \pm 7.1$ & $20.8 \pm 19.1$ & 0.112 \\
\hline ECMO & $2(3.3)$ & $1(10.0)$ & 0.375 \\
\hline CRRT & $3(5.0)$ & $1(10.0)$ & 0.468 \\
\hline Reoperation & $1(1.7)$ & $1(10.0)$ & 0.267 \\
\hline Intra-tracheal reintubation & $1(1.7)$ & $1(10.0)$ & 0.267 \\
\hline Infection & $0(0.0)$ & $1(10.0)$ & 0.142 \\
\hline Atrial thrombosis & $0(0.0)$ & $0(0.0)$ & 1.000 \\
\hline Acute rejection & $1(1.7)$ & $0(0.0)$ & 1.000 \\
\hline 30-day mortality & $1(1.7)$ & $1(10.0)$ & 0.267 \\
\hline \multicolumn{4}{|l|}{ Echocardiography } \\
\hline LVEF (\%) & $57.5 \pm 3.6$ & $58.2 \pm 2.7$ & 0.559 \\
\hline LAD (mm) & $33.9 \pm 3.1$ & $34.8 \pm 6.0$ & 0.653 \\
\hline LVDD (mm) & $42.5 \pm 3.2$ & $41.3 \pm 3.9$ & 0.291 \\
\hline MR (grade 0-4) & $0.2 \pm 0.5$ & $0.1 \pm 0.3$ & 0.395 \\
\hline TR (grade 0-4) & $0.4 \pm 0.7$ & $0.1 \pm 0.3$ & 0.029 \\
\hline
\end{tabular}

Values are expressed as mean \pm standard deviation or $\mathrm{n}(\%)$. ICU, intensive care unit; ECMO, extracorporeal membrane oxygenation; CRRT, continuous renal replacement; MODS, multiple organ dysfunction syndrome; LVEF, left ventricular ejection fraction; LAD, left atrium dimension; LVDD, left ventricular end diastolic dimension; MR, mitral regurgitation; TR, tricuspid regurgitation.

CPB time are further prolonged, the balance between the anticoagulation and the procoagulant system is further disrupted, leading to a longer hemostasis time and blood product transfusion (9). Therefore, careful separation of the adhesion tissue during surgery is the key to the operation's success for recipients with a history of prior cardiac surgery. 

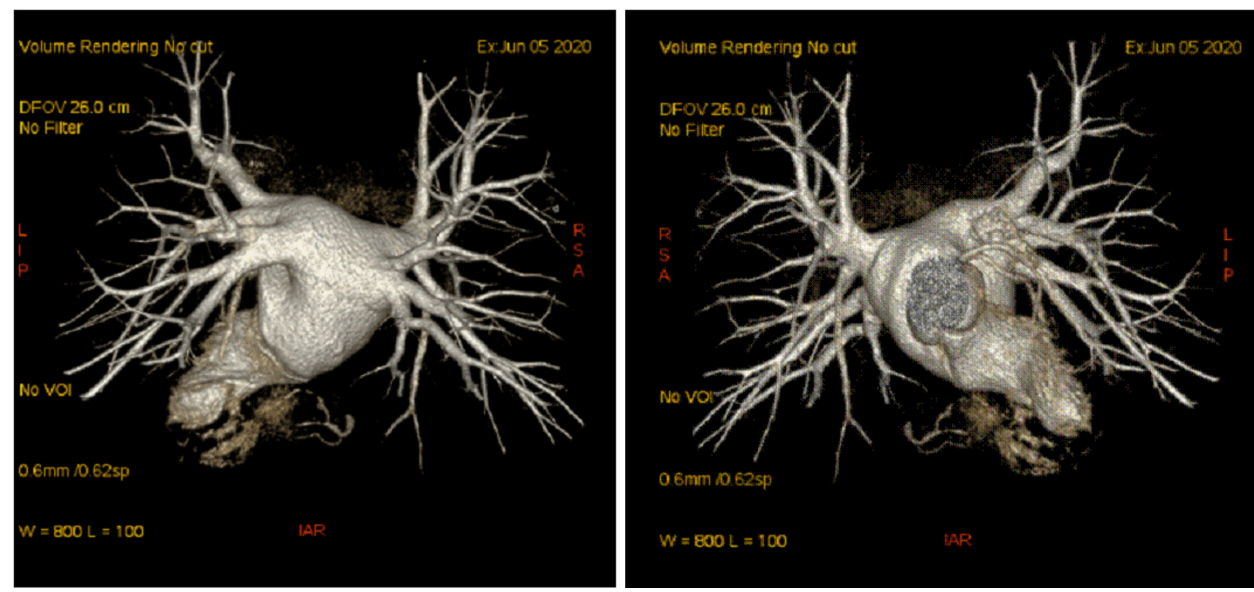

Figure 2 Postoperative CTA of left atrium and pulmonary veins. CTA and 3D reconstruction of an HTx recipient with four-corners traction bicaval anastomosis technique illustrated ideal and physiologic normal morphological structure of left atrial and pulmonary veins without significant kinking, distortion or dilation. No left atrial thrombosis was detected as well. CTA, computed tomography angiography; HTx, heart transplantation.

Table 4 Postoperative mid-term survival analysis

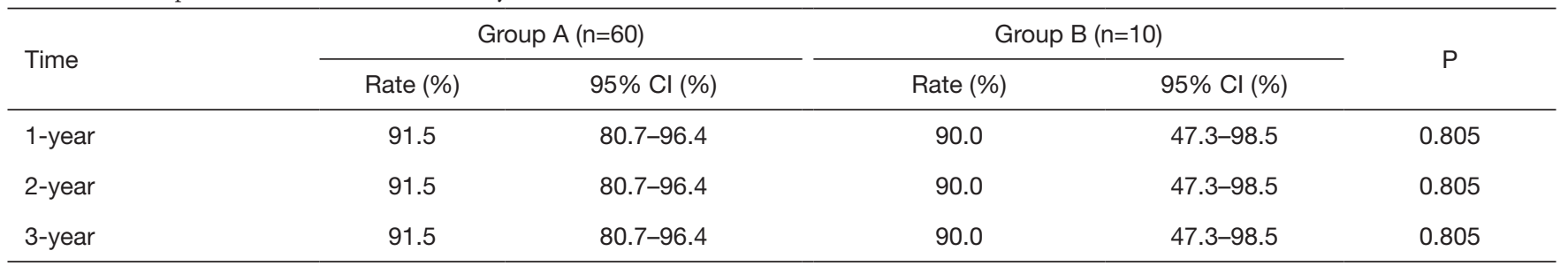

Values are expressed as mean \pm standard deviation. $\mathrm{Cl}$, confidence interval.

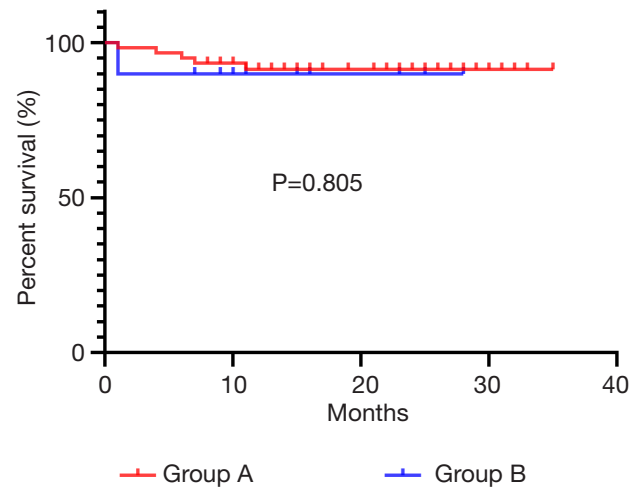

Figure 3 Kaplan-Meier plots of overall survival between 2 groups. There was no statistically difference in the post-operative midterm survival rate between patients transplanted without prior cardiac surgery (group A) and patients transplanted after prior cardiac surgery (group B) $(\mathrm{P}>0.05)$.
Sufficient preoperative organ function evaluation and intraoperative organ protection are also essential to prevent ischemia damage caused by the prolonged operation time.

\section{Femoral artery intubation to ensure surgical safety}

Through the femoral artery and vena cava cannulation, the CPB method is a safer option for recipients' intraoperative surgery with a prior cardiac surgical history (10). Due to the dense adhesion between the heart and sternum caused by the prior cardiac surgery, the transplantation method of the mid-sternal incision can easily cut the heart and aorta, and in severe cases, the patient is in shock due to rapid, massive bleeding. Moreover, this method of peripheral vascular intubation also provides more chest operation space for transplantation. 


\section{Four-corners traction prevents donor beart distortion}

In the early years of HTx, the biatrial anastomosis method was first adopted in 1960, but the high incidence of severe mitral valve dilation and continuous regurgitation of atrioventricular valves was noticed with the popularization of this method (11). Subsequently, bicaval anastomosis and other modified methods were introduced to reduce the above complications. However, postoperative complications such as donor heart distortion, atrial thrombosis, and anastomotic hemorrhage still exist (12), which is why we propose the novel modified bicaval anastomosis technique of HTx. Riberi and his colleagues found, through transesophageal echocardiography, that the surface of the left atrium was expanded to $33 \pm 4 \mathrm{~cm}$ in the classical transplantation anastomosis method (13). Due to the fourcorner traction technique applied in this study, the left atrial cuff was shaped into a quadrilateral that contributed to proportionally controlling each edge distance of the left atrial cuff to precisely match the left atrial cuff of the recipient. The postoperative CTA results illustrated an ideal and physiologically normal morphological structure of the left atrial and pulmonary veins without significant distortion or dilation after the novel modified anastomosis technique. Before discharge, the average LVEF in groups A and $\mathrm{B}$ reached $57.5 \% \pm 3.6 \%$ and $58.2 \% \pm 2.7 \%$, respectively, and there were almost no cases of mitral and tricuspid regurgitation observed.

\section{Continuous everting suture prevent thrombosis}

A previous study reported that a left atrial thrombus was found in 11 of 75 patients (15\%) who had undergone the biatrial anastomosis method (14). The reasons for the formation of atrial thrombosis after HTx are still a matter of debate. Studies have found that, due to conventional continuous suture techniques, exposure of the collagen fibrils and fat under the vascular endothelium to the blood in the heart cavity activates platelets and the intrinsic coagulation cascade leading to the formation of thrombus. The protruding suture can also create a niche just above the left atrial appendage where stasis and thrombosis are favored $(15,16)$. No cases of postoperative atrial thrombosis or cerebral embolism were observed during hospitalization in this study, and we attribute this to the complete everting suture method used in the HTx operation. The complete everting suture technique used in this study maintains the endocardium's integrity and avoids platelet activation caused by exposure of the collagen of the atrial wall to the blood in the heart cavity. And the consistent eversion of the atrial intima causes the continuous laminar flow of blood in the atrium cavity and reduces the occurrence of turbulence at the anastomosis when blood flow is resumed.

\section{PRA and acute rejection}

Studies have reported that the recipient's preoperative PRA level is associated with early rejection after HTx (17). A study conducted by Ibrahim et al., demonstrated that post-listing transfusions were associated with a $28 \%$ decreased likelihood of undergoing transplantation and a more than 4-fold increased risk of death (18). Preoperative blood transfusion is an independent risk factor for elevated PRA. Multivariate analysis has indicated that transfusion and more than five blood product transfused units were related to either class 1 or class 2 PRA positivity avoidance of the blood transfusion-associated antigens improving transplantation outcomes (19). Among the recipients with a history of prior cardiac surgery in this study, $90 \%$ had a blood transfusion history before transplantation compared to $0 \%$ in the recipients without prior cardiac surgery. This may be the reason why their average level of PRA is higher than that of recipients without a prior history of blood transfusion, although there was no statistical difference. This phenomenon implies recipients with prior cardiac surgery history may be more susceptible to early postoperative rejection than those without prior cardiac surgery. However, this possibility did not eventuate in our study. There may be three reasons for this result: First, all recipients in our medical center undergo CDC experiments while undergoing PRA screening, and the CDC results of all recipients who had undergone HTx in this study were negative; second, a satisfactory immunosuppression protocol was adopted in this study: for all recipients, this involved preoperative induction with Basiliximab, combined with intraoperative and postoperative methylprednisolone shock therapy, and finally triple immunosuppressive therapy. Furthermore, antithymocyte globulin (ATG) medicine is used to replace Basiliximab in treating PRA-positive recipients to enhance the immunosuppression effect, and plasma exchange combined with a rituximab regimen can reduce the HLA antibody titer; thirdly, the group with a history of cardiac surgery in this study constituted a limited sample size. We can see the statistical difference in PRA between the two groups through the subsequent longer follow-up period. Therefore, it is important to assess 
and screen suitable transplant recipients before surgery carefully. Meanwhile, we should pay attention to whether patients have symptoms and signs of heart failure after $\mathrm{HTx}$, monitor whether the concentration of anti-rejection drugs decreases, and whether myocardial enzymes as well as brain natriuretic peptide increase and judge the possibility of immune rejection combined with comprehensive factors such as electrocardiogram and cardiac ultrasound changes. If the above medical evidence appears, an endomyocardial biopsy (EMB) should be performed to confirm whether AR has occurred. In this study, a case of AR was observed, and the EMB showed extensive myocardial interstitial edema with diffuse infiltration of lymphocytes and epicardial cellulose-like necrosis. The patient was graded as $3 \mathrm{R}$ according to the 2004 classification of cardiac rejection and finally died of MODS due to AR. When AR is suspected, it is necessary to strengthen immunotherapy such as highdose glucocorticoid shock therapy and ATG combined with gamma globulin shock therapy.

\section{Early and mid-term survival}

A meta-analysis and several studies showed similar 30-day, 1-, 2-, and 5-year mortality rates between both groups (20). Another study found that survival was reduced in a prior cardiac surgery group $>4$ years after HTx (21). Some studies have tried to show that previous cardiac surgery history may be an independent risk factor for long-term death after HTx. In this study, our follow-up 35 months after surgery revealed that the 30-day mortality, and 1-, 2-, and 3-year survival rates were comparable between the prior cardiac surgery group and the non-prior cardiac surgery group. Careful preoperative evaluation and strict postoperative organ function monitoring can protect transplant recipients with previous cardiac surgery history to achieve a similar early and mid-term survival outcome compared to that of recipients without a history of cardiac surgery. Also, the application of the four-corner traction bicaval anastomosis technique for HTx and a satisfactory immunosuppression protocol contribute to the prognosis of recipients.

\section{Limitations}

It cannot be denied that there are some limitations in this study: The sample size of patients who underwent the HTx procedure especially those with a previous history of cardiac surgery is relatively small. Additionally, the retrospective, observational nature of the study is also a limitation of this research. However, considering the large proportion of recipients worldwide with a history of previous heart surgery, this study's results are important. In the future, we aim to conduct studies with larger sample sizes and a 10-year follow-up to compare the clinical outcomes between recipients transplanted with and without a history of prior cardiac surgery to validate the current findings.

\section{Acknowledgments}

This project is attributed to the Department of Cardiovascular Surgery, Renmin Hospital of Wuhan University.

Funding: This study was supported by grants from National Natural Science Foundation of China (82070481).

\section{Footnote}

Reporting Checklist: The authors have completed the TREND reporting checklist Available at http://dx.doi. org/10.21037/atm-21-317

Data Sharing Statement: Available at http://dx.doi. org/10.21037/atm-21-317

Peer Review File: Available at http://dx.doi.org/10.21037/ atm-21-317

Conflicts of Interest: All authors have completed the ICMJE uniform disclosure form (available at http://dx.doi. org/10.21037/atm-21-317). The authors have no conflicts of interest to declare.

Ethical Statement: The authors are accountable for all aspects of the work in ensuring that questions related to the accuracy or integrity of any part of the work are appropriately investigated and resolved. All trial protocols are conducted in accordance with the Declaration of Helsinki (as revised in 2013) and approved by the Human Research Ethics Committees of Renmin Hospital of Wuhan University (No. WDRY2020-K230). Our study complies with the International Society for Heart and Lung Transplantation ethics statement. Written informed consent was obtained from all patients or their guardians.

Open Access Statement: This is an Open Access article distributed in accordance with the Creative Commons Attribution-NonCommercial-NoDerivs 4.0 International 
License (CC BY-NC-ND 4.0), which permits the noncommercial replication and distribution of the article with the strict proviso that no changes or edits are made and the original work is properly cited (including links to both the formal publication through the relevant DOI and the license). See: https://creativecommons.org/licenses/by-nc-nd/4.0/.

\section{References}

1. Huenges K, Panholzer B, Fritzsche K, et al. Over Ten Years of Experience with a Modified Right Atrial Anastomosis in Orthotopic Heart Transplantation: Follow-up and Comparison with the Biatrial and Bicaval Technique. Thorac Cardiovasc Surg 2018;66:410-6.

2. Kittleson MM, Kobashiqawa JA. Cardiac transplantation: Current outcomes and contemporary controversies. JACC Heart Fail 2017;5:857-68.

3. Kansara P, Czer L, Awad M, et al. Heart transplantation with and without prior sternotomy: analysis of the United Network for Organ Sharing database. Transplant Proc 2014;46:249-55.

4. Aziz T, Burgess M, Rahman A, et al. Early and longterm results of heart transplantation after previous cardiac surgery. Eur J Cardiothorac Surg 2000;17:349-54.

5. Auerbach SR, Smith JK, Gralla J, et al. Graft survival is better without prior surgery in cardiac transplantation for functionally univentricular hearts. J Heart Lung Transplant 2012;31:987-95.

6. Maltais S, Haglund NA, Davis ME, et al. Outcomes After Concomitant Procedures with Left Ventricular Assist Device Implantation: Implications by Device Type and Indication. ASAIO J 2016;62:403-9.

7. Wellmann P, Herrmann FE, Hagl C, et al. A Single Center Study of 1,179 Heart Transplant Patients-Factors Affecting Pacemaker Implantation. Pacing Clin Electrophysiol 2017;40:247-54.

8. Kokkinos C, Athanasiou T, Rao C, et al. Does re-operation have an effect on outcome following HTx? Heart Lung Circ 2007;16:93-102.

9. Scrascia G, Rotunno C, Simone S, et al. Acute kidney injury in high-risk cardiac surgery patients: roles of inflammation and coagulation. J Cardiovasc Med (Hagerstown) 2017;18:359-65.

10. Saadat S, Schultheis M, Azzolini A, et al. Femoral cannulation: a safe vascular access option for cardiopulmonary bypass in minimally invasive cardiac surgery. Perfusion 2016;31:131-4.

11. Beniaminovitz A, Savoia MT, Oz M, et al. Improved atrial function in bicaval versus standard orthotopic techniques in cardiac transplantation. Am J Cardiol 1997;80:1631-5.

12. Maning J, Blumer V, Hernandez G, et al. Bicaval vs biatrial anastomosis techniques in orthotopic heart transplantation: An updated analysis of the UNOS database. J Card Surg 2020;35:2242-7.

13. Riberi A, Ambrosi P, Habib G, et al. Systemic embolism: a serious complication after cardiac transplantation avoidable by bicaval technique. Eur J Cardiothorac Surg 2001;19:307-11; discussion 311-2.

14. Derumeaux G, Habib G, Schleifer DM, et al. Standard orthotopic heart transplantation versus total orthotopic heart transplantation. A transesophageal echocardiography study of the incidence of left atrial thrombosis. Circulation 1995;92:II196-201.

15. Castedo E, Burgos R, Cañas A, et al. Left atrial thrombosis after heart transplantation. Cardiovasc Surg 2003;11:247-9.

16. Escribano Subias P, Gómez-Sánchez MA, Fernández Casares S, et al. Incidence and dynamic behavior of spontaneous echocardiographic contrast and atrial thrombi in the transplanted heart. Rev Esp Cardiol 2001;54:1055-60.

17. Bynum JP, Zachary A, Ness PM, et al. Transfusion of leukoreduced blood products and risk of antibodymediated rejection of renal allografts. Transfusion 2018;58:1951-7.

18. Ibrahim HN, Skeans MA, Li Q, Ishani A, Snyder JJ. Blood transfusions in kidney transplant candidates are common and associated with adverse outcomes. Clin Transplant 2011;25:653-9.

19. Can Ö, Gökçe AM, Canbakan M, et al. Evaluation of Pre-Transplant Panel Reactive Antibody Levels and Sensitization: A Single-Center Study. Ann Transplant 2016;21:577-81.

20. Awad M, Czer LS, Mirocha J, et al. Prior sternotomy increases the mortality and morbidity of adult HTx. Transplant Proc 2015;47:485-97.

21. Gaffey AC, Phillips EC, Howard J, et al. Prior Sternotomy and Ventricular Assist Device Implantation Do Not Adversely Impact Survival or Allograft Function After HTx. Ann Thorac Surg 2015;100:542-9.

Cite this article as: Shi F, Ren Z, Zhang M, Wang Z, Wu Z, Hu X, Hu Z, Wu H, Ren W, Li L, Ruan Y, Hu R. Effect of novel bicaval anastomosis technique for transplantation with and without prior cardiac surgery history. Ann Transl Med 2021;9(13):1064. doi: 10.21037/atm-21-317 